\title{
Simultaneously constraining the astrophysics of reionisation and the epoch of heating with 21CMMC
}

\author{
Bradley Greig and Andrei Mesinger \\ Scuola Normale Superiore, Piazza dei Cavalieri 7, I-56126 Pisa, Italy \\ email: brad.s.greig@gmail.com
}

\begin{abstract}
We extend our MCMC sampler of 3D EoR simulations, 21CMMC, to perform parameter estimation directly on light-cones of the cosmic $21 \mathrm{~cm}$ signal. This brings theoretical analysis one step closer to matching the expected $21-\mathrm{cm}$ signal from next generation interferometers like HERA and the SKA. Using the light-cone version of 21CMMC, we quantify biases in the recovered astrophysical parameters obtained from the $21 \mathrm{~cm}$ power spectrum when using the co-eval approximation to fit a mock 3D light-cone observation. While ignoring the light-cone effect does not bias the parameters under most assumptions, it can still underestimate their uncertainties. However, significant biases $(\sim$ few $-10 \sigma)$ are possible if all of the following conditions are met: (i) foreground removal is very efficient, allowing large physical scales $\left(k \sim 0.1 \mathrm{Mpc}^{-1}\right)$ to be used in the analysis; (ii) theoretical modelling is accurate to $\sim 10$ per cent in the power spectrum amplitude; and (iii) the $21 \mathrm{~cm}$ signal evolves rapidly (i.e. the epochs of reionisation and heating overlap significantly
\end{abstract}

Keywords. galaxies: high-redshift, intergalactic medium, cosmology: theory, early universe

\section{Introduction}

The most promising observable for investigating the epoch of reionisation (EoR) and cosmic dawn is the $21 \mathrm{~cm}$ spin-flip transition of neutral hydrogen. This is observed in offset against the cosmic microwave background, as the $21 \mathrm{~cm}$ brightness temperature, $\delta T_{\mathrm{b}}$ (see e.g. Furlatetto et al. 2006). Owing to its spatial and frequency (redshift) dependence, the $21 \mathrm{~cm}$ signal will reveal a full three dimensional image of the intergalactic medium (IGM). The signal is sensitive to the IGM's ionisation state, enabling an exploration of the EoR morphology and inferences on the ionising sources. It is also sensitive to its thermal state, allowing a study of high-energy processes in the first galaxies, thought to dominate the heating of the IGM (epoch of heating; EoH) which precedes the EoR.

Numerous radio experiments seek the first detection of this $21 \mathrm{~cm}$ signal. However, only the Hydrogen Epoch of Reionization Array (HERA) (DeBoer et al. 2017) and Square Kilometre Array (SKA) (Mellema et al. 2013) will have sufficient sensitivity to achieve a measurement of the $21 \mathrm{~cm}$ power spectrum (PS) spanning the EoR and EoH. These experiments will generate an enormous volume of data, comfortably entering the realms of 'Big Data'. This necessitates huge efforts to develop sophisticated methods for theoretical interpretation and astrophysical parameter forecasting. We developed one such approach with our Monte Carlo Markov Chain EoR analysis tool, 21CMMC (Greig \& Mesinger 2015, 2017), which performs on-the-fly sampling of 3D EoR/CD simulations using 21cmFAST (Mesinger \& Furlanetto, Mesinger et al. 2011).

Typically the co-evolution ('co-eval') approximation is assumed. That is, simulation cubes have the same redshift along the line-of-sight. This is despite the fact the line-ofsight signal varies, producing the so-called observed light-cone. Light-cone effects have 
been shown to modify the large scales of the 21cm PS (Datta et al. 2012, 2014; La Plante et al. 2014; Ghara et al. 2015; Mondal et al. 2017), resulting in an increase/decrease in the PS amplitude in the initial/late stages of the EoR relative to the co-eval PS. While it is clear these light-cone effects impact the $21 \mathrm{~cm}$ PS, it is unclear whether this effect leads to any significant biases in the recovered astrophysical parameters. Therefore, in this work we have extended 21CMMC to perform parameter inference directly on the sampled 3D light-cones. To quantify the effect, we consider two reionisation histories, the FAINT GALAXIES and BRIGHT GALAXIEs models of Greig \& Mesinger (2017). These constitute slow and fast reionisation histories, respectively, are consistent with existing observational constraints and should bracket the extent of the bias recovered from astrophysical parameter forecasting.

\section{Overview}

Below, we summarise the changes made to $21 \mathrm{CMMC}$, the astrophysical parameter set and our 21CMMC setup for exploring the impact of the light-cone effects.

Extensions to $21 C M M C$. In addition to the $21 \mathrm{~cm}$ light-cone, a secondary observational effect is redshift-space distortions. Observations are made in redshift space rather than real space, therefore the observed $21 \mathrm{~cm}$ signal is distorted from the true $21 \mathrm{~cm}$ signal by line-of-sight peculiar velocities. In this work, we include redshift space distortions to our observed light-cones. Finally, when calculating the $21 \mathrm{~cm}$ light-cone, we use the full expression for the $21 \mathrm{~cm}$ opacity rather than the optically thin approximation.

Astrophysical Parameter Set. In total, 21CMMC has six astrophysical parameters, which we briefly outline below (refer to Greig \& Mesinger 2017 for detailed descriptions).

- $\zeta$ - the UV ionising efficiency of high-z galaxies. This describes the amount of ionising photons escaping into the IGM from the ionising sources. It controls the timing of reionisation, with larger values shifting reionisation to earlier times while also shortening its duration. We adopt a flat prior over the fiducial range of $\zeta \in[10,250]$.

- $R_{\mathrm{mfp}}$ - maximum ionising photon horizon. Photon sinks (e.g. Lyman limit systems) and associated recombinations limit the extent to which photons can propagate through the ionised IGM. This effect is approximated with a maximum horizon through the ionised IGM, $R_{\mathrm{mfp}}$. It has a minimal impact on reionisation, potentially slowing down the end stages when the typical ionised regions exceed $R_{\mathrm{mfp}}$. We adopt a flat prior over $R_{\mathrm{mfp}} \in[5,25] \mathrm{cMpc}$

- $T_{\mathrm{vir}}^{\mathrm{min}}$ : minimum virial temperature of star-forming haloes. Galaxies hosted in halos with virial temperatures above $T_{\text {vir }}^{\min }$ are assumed to have a constant ionising luminosity to halo mass, while galaxies below are assumed to have negligible star-formation (e.g. negative feedback and/or difficulty in cooling). We assume star-forming galaxies to be the source of all radiation fields governing the cosmic $21-\mathrm{cm}$ signal, therefore $T_{\mathrm{vir}}^{\mathrm{min}}$ impacts the timing and source bias of all cosmic epochs. Increasing $T_{\mathrm{vir}}^{\mathrm{min}}$ results in later, more rapid evolution of the $21 \mathrm{~cm}$ signal. We take a flat prior across $T_{\text {vir }}^{\min } \in\left[10^{4}, 10^{6}\right] \mathrm{K}$.

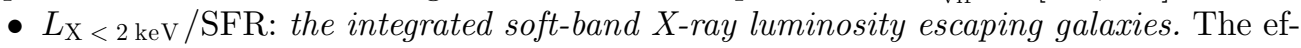
ficiency of X-rays at heating the IGM preceding the EoR is driven by the total integrated soft-band $(<2 \mathrm{keV})$ luminosity per star formation rate (SFR) escaping the host galaxies. It regulates the timing of the EoH (analogous to the role of $\zeta$ for the EoR). We adopt a flat prior over the range, $\log _{10}\left(L_{\mathrm{X}<2 \mathrm{keV}} / \mathrm{SFR}\right) \in[38,42] \mathrm{erg} \mathrm{s}^{-1} M_{\odot}^{-1}$ yr.

- $E_{0}$ : X-ray energy threshold for self-absorption by the host galaxies. The interstellar medium of high- $z$ galaxies can absorb soft X-ray photons, attenuating the emergent Xray profile, and limiting IGM heating. At fixed soft-band luminosities, increasing $E_{0}$ 
Table 1. Summary of the faint galaxies (top) and Bright galaxies (bottom) models, and the median recovered values (and associated 16th and 84th percentile errors). We assume a $1000 \mathrm{hr}$ observation with HERA, sampling the mock observation with the light-cone (LC) or co-eval approximation (co-eval). Note, we anticipate similar behaviour for the SKA (see text).

\begin{tabular}{|c|c|c|c|c|c|c|c|c|}
\hline $\begin{array}{c}\text { HERA } 331 \\
\text { Model }\end{array}$ & $\zeta$ & $\begin{array}{l}R_{\mathrm{mfp}} \\
{[\mathrm{Mpc}]}\end{array}$ & $\begin{array}{c}\log _{10}\left(T_{\mathrm{vir}}^{\mathrm{min}}\right) \\
{[\mathrm{K}]}\end{array}$ & $\begin{array}{l}\log _{10}\left(\frac{L \mathrm{X}<2 \mathrm{keV}}{\mathrm{SFR}}\right) \\
{\left[\mathrm{erg} \mathrm{s}^{-1} M_{\odot}^{-1} \mathrm{yr}\right]}\end{array}$ & $\begin{array}{c}E_{0} \\
{[\mathrm{keV}]}\end{array}$ & $\alpha_{X}$ & $\tau_{\mathrm{e}}$ & $\chi_{\mathrm{min}}^{2}$ \\
\hline FAINT & 30.0 & 15.0 & 4.70 & 40.0 & 0.5 & 1.0 & 0.059 & - \\
\hline $\mathrm{LC}$ & $32.20_{-2.08}^{+2.68}$ & $14.86_{-0.86}^{+0.78}$ & $4.74_{-0.03}^{+0.04}$ & $40.06_{-0.06}^{+0.05}$ & $0.50_{-0.14}^{+0.09}$ & $0.63_{-0.92}^{+1.17}$ & - & 8.60 \\
\hline Co-eval & $31.28_{-1.87}^{+2.32}$ & $14.77_{-0.80}^{+0.81}$ & $4.72_{-0.03}^{+0.04}$ & $40.03_{-0.05}^{+0.05}$ & $0.42_{-0.05}^{+0.04}$ & $1.15_{-0.44}^{+0.52}$ & - & 18.14 \\
\hline BRIGHT & 200.0 & 15.0 & 5.48 & 40.0 & 0.5 & 1.0 & 0.056 & - \\
\hline $\mathrm{LC}$ & $196.01_{-17.04}^{+16.23}$ & $14.56_{-0.77}^{+0.85}$ & $5.48_{-0.01}^{+0.01}$ & $40.04_{-0.05}^{+0.05}$ & $0.54_{-0.07}^{+0.05}$ & $0.68_{-0.90}^{+0.95}$ & - & 14.34 \\
\hline Co-eval & $114.26_{-5.32}^{+5.72}$ & $22.88_{-1.16}^{+0.93}$ & $5.40_{-0.01}^{+0.01}$ & $39.92_{-0.02}^{+0.02}$ & $0.18_{-0.05}^{+0.04}$ & $0.15_{-0.08}^{+0.07}$ & - & 44.55 \\
\hline
\end{tabular}

hardens the emerging spectrum, resulting in more uniform heating. We take a flat prior over $E_{0} \in[0.1,1.5] \mathrm{keV}$.

- $\alpha_{\mathrm{X}}: X$-ray spectral energy index. The spectral index depends on the dominant physical process producing the X-ray photons. Increasing/decreasing $\alpha_{\mathrm{X}}$ results in more numerous soft/hard X-ray photons (when the soft-band luminosity is fixed), resulting in similar qualitative behaviour to that for decreasing/increasing $E_{0}$. We adopt a flat prior of $\alpha_{\mathrm{X}} \in[-1.0,3.0]$ covering a range of plausible X-ray spectral energy distributions.

21CMMC Setup. We generate mock light-cones from $600^{3} \mathrm{Mpc}^{3}$ co-eval cubes on a $400^{3}$ grid and analyse the light-cone signal by splitting the light-cone into equal comoving distance cubes and generating the $21 \mathrm{~cm}$ PS for each. Our light-cones cover $z=6$ $(\sim 200 \mathrm{MHz})$ to $z=26.8(\sim 50 \mathrm{MHz})$, consistent with the observing frequency of HERA and the SKA. For the 21CMMC runs, we sample $300^{3} \mathrm{Mpc}^{3}$ co-eval cubes on a $200^{3}$ grid, fitting the sampled $21 \mathrm{~cm}$ PS to the mock PS between $k=0.1-1.0 \mathrm{Mpc}^{-1}$. We also include a constant 10 per cent modelling uncertainty on the sampled $21 \mathrm{~cm}$ PS. For the co-eval approximation, the central redshift of the light-cone cube is used as the co-eval redshift. We assume a $1000 \mathrm{hr}$ observation with HERA, noting that we anticipate similar behaviour with the SKA owing to its similar performance in Greig \& Mesinger (2017).

\section{Implications}

In Table 1 we show the recovered astrophysical parameter constraints from the two models, and in Figure 1 we present the BRIGHT GALAXIES posterior distributions. We summarise the main findings for each model below.

FAINT GALAXIES. We recover no biases in the astrophysical parameters when ignoring the light-cone effects. However, we do find that the recovered precision of the astrophysical parameters in the EoH can be overestimated by more than a factor of two.

BRIGHT GALAXIES. We find strong biases $(\sim$ few $-10 \sigma)$ in the recovered parameters (see Figure 1) when ignoring the light-cone effect. These arise due to the rapidly evolving $21 \mathrm{~cm}$ signal along the line-of-sight, amplifying the large-scale variations in the $21 \mathrm{~cm}$ PS.

However, we caution that out setup was designed to maximise the potential effect: (i) we decreased our modelling uncertainty to 10 per cent; (ii) extended our fitting range to larger (more contaminated) scales (more optimistic about astrophysical foreground removal) and (iii) we considered the spherically averaged $21 \mathrm{~cm}$ PS, which is sub-optimal for a signal evolving along the line-of-sight (see e.g. the wavelet transform Trott 2016). 


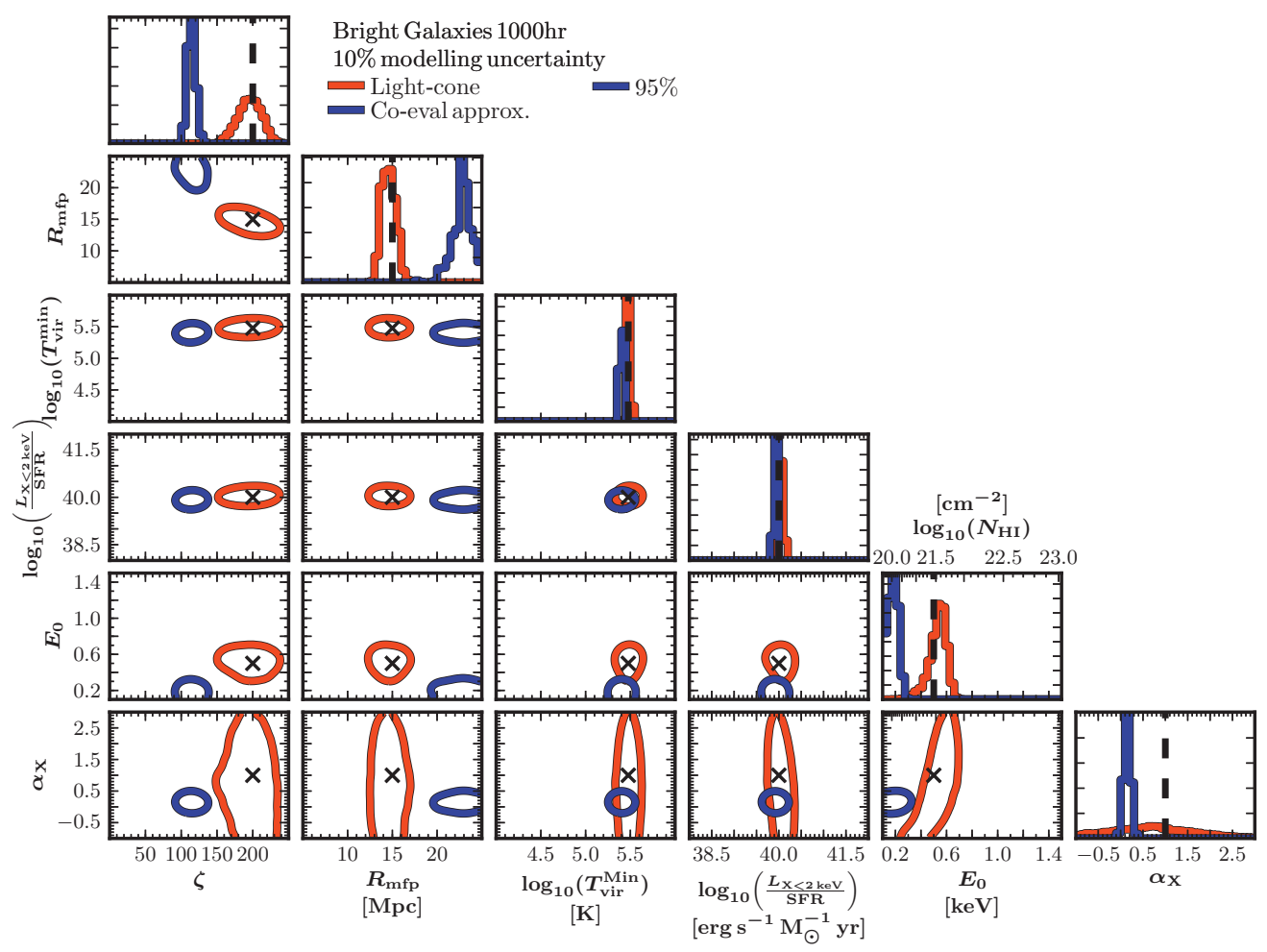

Figure 1. Recovered 1 and 2D marginalised posteriors for the BRIGHT GALAXIES mock observation, assuming $1000 \mathrm{hrs}$ observing with HERA. Different curves correspond to fitting the mock observation with the co-eval approximation (blue) and the light-cone (red). Contours are the 95th percentiles, with the black crosses (vertical lines) denoting the input model parameters.

Therefore, ignoring the light-cone effect can have a significant effect on the inferred astrophysical parameters, however, only under certain conditions.

\section{References}

Datta, K. K., Mellema, G., Mao, Y., Iliev, I. T., Shapiro, P. R., \& Ahn K. 2012, MNRAS, 424, 1877

Datta, K. K., Jensen, H., Majumdar, S., Mellema, G., Iliev, I. T., Mao, Y., Shapiro, P. R., \& Ahn K. 2014, MNRAS, 442, 1491

DeBoer, D. R., et al. 2017, PASP, 129, 5001

Furlanetto, S. R., Oh, S. P., \& Briggs F. H. 2006, Phys. Rep., 433, 181

Ghara, R., Datta, K. K., \& Choudhury, T. R. 2015, MNRAS, 453, 3143

Greig, B. \& Mesinger, A. 2015, MNRAS, 449, 4246

Greig, B. \& Mesinger, A. 2017, MNRAS, 472, 2651

Jensen, H., et al. 2013, MNRAS, 435, 460

La Plante, P., Battaglia, N., Natarajan, A., Peterson, J. B., Trac, H., Cen, R., \& Loeb, A. 2014, ApJ, 789, 31

Mellema, G., et al. 2013, Exp. Astron., 36, 235

Mesinger, A. \& Furlanetto, S. R. 2007, ApJ, 669, 663

Mesinger, A., Furlanetto, S. R., \& Cen R. 2011, MNRAS, 431, 955

Mondal, R., Bharadwaj, S., \& Datta, K. K. 2017, arXiv:1706.09449

Trott, C. M. 2016, MNRAS, 461, 126 